\title{
Atypical Evolution of Secondary Hemolytic Uremic Syndrome Defined as Paraneoplastic Syndrome under Eculizumab and Palbociclib Therapies
}

\author{
Quentin Perrier $^{a} \quad$ Johan Noble ${ }^{b} \quad$ Steven Grangéc, d Pierrick Bedouch ${ }^{a, e, f}$ \\ Rachel Tetaz $^{\mathrm{b}} \quad$ Lionel Rostaing ${ }^{\mathrm{b}}$ e \\ aDepartment of Clinical Pharmacy, Grenoble Alpes University Hospital, Grenoble, France; \\ ${ }^{b}$ Nephrology, Hemodialysis, Apheresis and Kidney Transplantation Department, Grenoble \\ Alpes University Hospital, Grenoble, France; 'Medical Intensive Care Unit, Rouen University \\ Hospital, Rouen, France; ${ }^{\mathrm{d} C e n t r e ~ d e ~ r e ́ f e ́ r e n c e ~ d e s ~ m i c r o a n g i o p a t h i e s ~ t h r o m b o t i q u e s ~(C N R-~}$ \\ MAT), AP-HP, Paris, France; ${ }^{\mathrm{G}}$ Grenoble Alpes University, Grenoble, France; ${ }^{\mathrm{f} G r e n o b l e ~ A l p e s}$ \\ University, CNRS TIMC-IMAG, UMR 5525, Grenoble, France
}

\section{Keywords}

Thrombotic microangiopathy · Breast cancer - Eculizumab - Atypical hemolytic uremic syndrome $\cdot$ Complement $\cdot$ Palbociclib $\cdot$ Metastases

\begin{abstract}
Thrombotic microangiopathy (TMA) is most of the time caused by thrombotic thrombocytopenic purpura or hemolytic uremic syndrome. A 60-year-old female was diagnosed in 2014 with mammary breast adenocarcinoma treated by several-line therapy: mastectomy, docetaxel, cyclophosphamide, radiotherapy, doxorubicine, and capecitabine. By mid-November, the patient was admitted to the hospital with regenerative, mechanical, and hemolytic anemia, schistocytes at $3 \%$, and thrombopenia $(99 \mathrm{G} / \mathrm{L})$, associated with high blood transfusion requirement. After 9 sessions of plasmapheresis, there was no significant improvement in the biological parameters, nor after 2 cycles of paclitaxel. The patient was then treated with eculizumab during 4 weeks, with a slight reduction in blood requirement, and simultaneously with palbociclib. Since being treated with palpociclib, she had a great reduction in blood requirement and a good clinical condition. To conclude, we reported an initial moderate improvement of paraneoplasm-related TMA syndrome under eculizumab therapy with a slight reduction in red blood cell requirement; however, palbociclib therapy achieved a very good response with a dramatic reduction in red blood cell requirement.
\end{abstract}

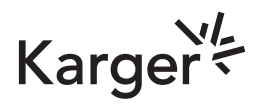




\section{Introduction}

Thrombotic microangiopathy (TMA) is defined as an association between mechanical hemolytic anemia, peripheral thrombopenia, and sometimes organ failure. Two main causes of TMA are thrombotic thrombocytopenic purpura and hemolytic uremic syndrome (HUS). Atypical HUS (aHUS) and typical HUS (tHUS) have been well described, whereas secondary HUS is heterogeneous and associated with various underlying conditions, that is, infection, solid- and bone-marrow transplantation, iatrogenesis, autoimmune disease, and malignancies. This definition was recently endorsed by international consensus [1, 2]. In particular, malignancy-related TMA can be defined as iatrogenic type 1, i.e., dose-dependent druginduced TMA (gemcitabine, mitomycin C, etc.), or as type 2, i.e., non-dose-dependent druginduced TMA (bevacizumab, sunitinib, etc.), and paraneoplastic syndromes [3] (Fig. 1).

Herein, we describe the atypical evolution of secondary HUS defined as a paraneoplastic syndrome in the setting of breast malignancy.

\section{Case Report}

A 60-year-old female was diagnosed in 2014 with mammary breast adenocarcinoma. As a first-line therapy, she underwent a mastectomy followed by 15 cycles of docetaxel/cyclophosphamide combined with letrozole hormone prophylaxis, and 25 radiotherapy sessions. Three years later, she presented with recurrence, i.e., metastasis to bones as well as to pleural and peritoneal spaces. The second line of treatment was 10 months of doxorubicin and hormone prophylaxis with fulvestrant plus 5 radiotherapy sessions. This treatment was followed by sustained therapy with capecitabine, and the bone metastases were managed with denosumab.

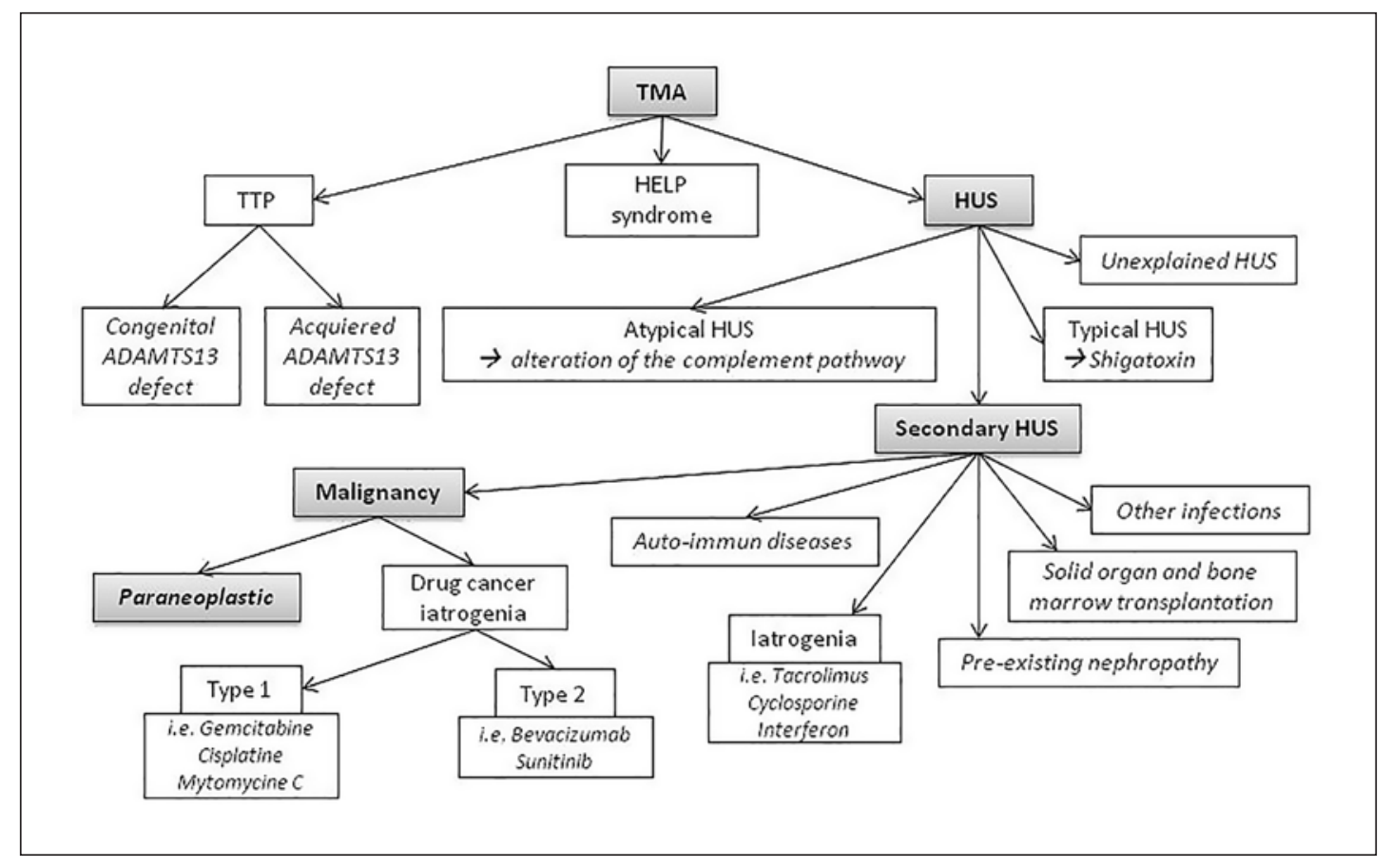

Fig. 1. TMA classification. TMA, thrombotic microangiopathy; TTP, thrombotic thrombocytopenic purpura; HUS, hemolytic uremic syndrome; ADAMT13, a disintegrin and metalloprotease with thrombospondin type I repeats-13. 




Fig. 2. Evolution of treatment, blood requirement, and biology of the patient during the period of interest. The arrow represents 1 red blood cell pack. The plasmapheresis took place over 10 days, then the patient received 2 cycles of paclitaxel, after which she received eculizumab during 4 weeks. Finally, she was concomitantly treated with palbociclib. C, cure.

By the end of October 2018, the patient initially presented with isolated hemolytic anemia associated with thrombopenia. Accordingly, capecitabine was stopped due to its potential iatrogenic effects [4], and therapy with $1 \mathrm{mg} / \mathrm{kg} /$ day of prednisolone was initiated. By midNovember, the patient was admitted to the hospital with regenerative, mechanical, hemolytic anemia (reticulocyte count $=306 \mathrm{G} / \mathrm{L}$; hemoglobin $=69 \mathrm{~g} / \mathrm{L}$ ), lactate dehydrogenase 9 times the upper normal value, haptoglobin below the titration level, and schistocytes at 3\%, associated with thrombopenia $(99 \mathrm{G} / \mathrm{L})$, but there was no renal failure. This situation was initially treated with 2-3 red blood cell transfusions per week. In addition, because this TMA was of unclear etiology, the patient was placed on plasmapheresis until the ADAMTS13 activity became available, that is, at $64 \%$.

There was no evidence of an autoimmune disorder, that is, no antibodies against $\mathrm{H}$ or I factors, exploration of complement activity showed no activation ( $\mathrm{CH} 50=150 \%$; $\mathrm{C} 3=1,260$ $\mathrm{mg} / \mathrm{L}[550-1,650] ;$ B factor = $315 \mathrm{mg} / \mathrm{L}$ [216-504]; C5 = $271 \mathrm{mg} / \mathrm{L}$ [120-220]), and no evidence of bacteremia and viremia (CMV, EBV, and hepatitis B, C and E viruses) in the analyses. After 9 sessions of plasmapheresis, there was no significant improvement in the biological parameters or any reduced need for blood transfusions. Thus, a third line of chemotherapy with paclitaxel was given for two 3-week cycles because secondary HUS due to paraneoplastic syndrome was suspected. However, because of persisting TMA and the fact that blood transfusion requirements were still high, the patient was in addition put on letrozole and palbociclib.

In parallel, and in order to block the complement pathway until third-line chemotherapy could control the TMA, the patient was given eculizumab ( $900 \mathrm{mg}$ per week). After 4 weeks of eculizumab therapy, the requirement for blood transfusions was decreased to 1 transfusion per week and $\mathrm{CH} 50$ was decreased to $14 \%$. After consulting the National Reference Center for TMA (CNR-MAT-Centre de référence des microangiopathies thrombotiques, Hôpital Saint-Antoine, Paris), and because of a lack of evidence that eculizumab could be effective in treating TMA-related paraneoplastic syndromes, that treatment was stopped. Since March 2019, the effectiveness of chemotherapy has enabled us to decrease the red 
blood cell transfusions to 2 per month. Figure 2 summarizes all changes in blood requirement, hemoglobin level, platelet count, and specific treatments since November 2018. Finally, the patient passed away in June 2020.

\section{Discussion}

We reported on a 60-year-old woman with metastatic breast cancer who presented with secondary HUS in the setting of paraneoplastic syndrome. This was successfully managed with palbociclib, a tyrosine kinase inhibitor, and we used eculizumab as a bridge to control TMA until palbociclib became effective in doing so.

One case of palbociclib-induced TMA has been previously described [5]; however, in our case, TMA had occurred well before palbociclib therapy was implemented. In addition, an association between palbociclib and letrozole enabled the patient to become stabilized. As a result, at 1 month after starting treatment, the requirement for blood transfusions had decreased to 1 transfusion every 2 weeks compared with 2-3 transfusions per week at the beginning.

The occurrence of secondary HUS in cancer patients is extremely low: no cases were reported among 2,046 patients in a randomized double-blinded study that focused on breast cancer and bone metastasis [6], even though some cases had previously been described [7, 8]. Although paraneoplastic TMA is rare, it needs a rapid diagnosis as it is associated with a high mortality rate [9]. However, in such a setting, it is important to know whether costly TMA therapies such as plasmapheresis, rituximab, or eculizumab can be implemented. At that point, we must balance the patient's life expectancy against the risk inferred by such therapies, for example, infectious complications, and the benefits for improving TMA.

Thus, before diagnosing paraneoplastic syndrome, other differential diagnoses must be eliminated. In cancer patients, the combination of anemia and thrombopenia can have different etiologies. Firstly, this might be related to inflammatory processes or to concomitant infections [3]. Secondly, disseminated intravascular coagulation must be ruled out; fibrinogen and D-dimer levels were normal in our patient [4]. Thirdly, thrombotic thrombocytopenic purpura has to be dismissed; it could be eliminated in our case because ADMST13 activity was normal, but we had the result about 10 days after diagnosing TMA. Finally, because (1) complement activity was normal, (2) there was neither renal dysfunction nor evidence for any underlying autoimmune disease, and (3) the patient had received no TMA-inducing drug, we consequently made the diagnosis of paraneoplastic syndrome. Moreover, this diagnosis was supported by other factors, the patient's age, the presence of adenocarcinoma with metastases, and the absence of improvement under plasma exchange therapy.

Eculizumab, a C5 inhibitor, is commonly used for aHUS and paroxysmal nocturnal hemoglobinuria [10]. Some success has been achieved when secondary HUS was associated with cancer and was described as malignancy iatrogenic type $1[8,11]$. The use of eculizumab in paraneoplastic syndrome is not recommended, because of its cost; in addition, cancer patients may have a poor clinical status, rapid evolution (weeks to days), and fatal issue[3]. However, for our patient, because of her very good clinical condition, in order to minimize blood transfusions and to provide bridging until palbociclib therapy was efficient, eculizumab was initiated. Unfortunately, its effectiveness was not great, as the patient still needed 1 red blood cell transfusion per week, and so treatment was stopped. We can suppose that a month of eculizumab treatment may have given enough time for palbociclib to achieve efficacy and control the neoplastic processes.

To conclude, we reported an initial moderate improvement of paraneoplasm-related TMA syndrome under eculizumab therapy with a slight reduction in red blood cell requirement;

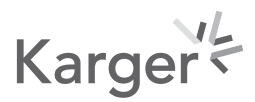


however, palbociclib therapy achieved a very good response with a dramatic reduction in red blood cell requirement.

\section{Statement of Ethics}

Our research was conducted ethically in accordance with the World Medical Association Declaration of Helsinki, and written informed consent was obtained from the patient's next of kin for publication of this case report and any accompanying images.

\section{Conflict of Interest Statement}

The authors have no conflicts of interest to declare.

\section{Funding Sources}

No funding was received.

\section{Author Contributions}

Q. Perrier contributed to acquisition of data and writing of the manuscript. J. Noble, S. Grangé, P. Bedouch, R. Tetaz, and L. Rostaing provided supervision and mentorship. All the authors approved the final manuscript.

\section{References}

1 Loirat C, Fakhouri F, Ariceta G, Besbas N, Bitzan M, Bjerre A, et al. An international consensus approach to the management of atypical hemolytic uremic syndrome in children. Pediatr Nephrol. 2016;31(1):15-39.

2 Le Clech A, Simon-Tillaux N, Provôt F, Delmas Y, Vieira-Martins P, Limou S, et al. Atypical and secondary hemolytic uremic syndromes have a distinct presentation and no common genetic risk factors. Kidney Int. 2019; 95(6):1443-52.

3 Kheder El-Fekih R, Deltombe C, Izzedine H. [Thrombotic Microangiopathy and cancer]. Nephrol Ther. 2017; 13(6):439-47.

4 Anastasiou M, Chalandon Y, Anchisi S. Anemie hemolytique dans le contexte de cancer. Rev Med Suisse. 2018; 14(607):1021-7.

5 Raiss H, Péron J, Tartas S, Trillet-Lenoir V, Freyer G, Errihani H. Palbociclib-induced thrombotic microangiopathy in metastatic breast cancer patient surviving for 18 years: case report and review of the literature. Clin Breast Cancer. 2018;18(3):e263-6.

6 Lechner K, Obermeier HL. Cancer-related microangiopathic hemolytic anemia: clinical and laboratory features in 168 reported cases. Medicine (Baltimore). 2012;91(4):195-205.

7 Izzedine H, Perazella MA. Thrombotic microangiopathy, cancer, and cancer drugs. Am J Kidney Dis. 2015; 66(5):857-68.

8 Hausberg M, Felten H, Pfeffer S. Treatment of chemotherapy-induced thrombotic microangiopathy with eculizumab in a patient with metastatic breast cancer. Case Rep Oncol. 2019;12(1):1-6.

9 Anastasiou M, Chalandon Y, Anchisi S. Anemie hemolytique dans le contexte de cancer. Rev Med Suisse. 2018; 14(607):1021-7.

10 Salant DJ. Targeting complement C5 in atypical hemolytic uremic syndrome. J Am Soc Nephrol. 2011;22(1): 7-9.

11 Martin K, Roberts V, Chong G, Goodman D, Hill P, Ierino F. Eculizumab therapy in gemcitabine-induced thrombotic microangiopathy in a renal transplant recipient. Oxf Med Case Reports.2019;2019(6):omz048.

\section{Karger ${ }^{\prime}=$}

